REVISTA UNIVERSIDAD DE GUAYAQUIL Vol. 132 No. 1-2021 (enero-junio) ISSN: 2477-913X e-ISSN 2806-5751

\title{
Motivación, cultura y creatividad elementos claves para generar emprendimientos en las instituciones de educación superior.
}

Motivation, culture and creativity key elements to generate entrepreneurship in higher education institutions.

\author{
Magdalena Rosario Huilcapi Masacon \\ Martha Mazacón Gómez \\ Luis Caicedo Hinojosa
}

Fecha de recepción: 03 de noviembre de 2020

Fecha de aceptación: 21 de diciembre de 2020 


\title{
Motivación, cultura y creatividad elementos claves para generar emprendimientos en las instituciones de educación superior.
}

\author{
Motivation, culture and creativity key elements to generate entrepreneurship in \\ higher education institutions. \\ Magdalena Rosario Huilcapi Masacon ${ }^{1}$, Martha Mazacón Gómez ${ }^{2}$, Luis Caicedo Hinojosa ${ }^{3}$ \\ Como citar: Huilcapi, M., Masacon, M, Caicedo, L. (2021). Motivación, cultura y creatividad elementos claves para generar emprendimientos en las \\ instituciones de educación superior, Revista Universidad de Guayaquil. 132(1), 41-47. DOI: https://doi.org/10.53591/rug.v132i1.1359
}

\section{RESUMEN}

Este trabajo se centra en la revisión de la base científica que tiene la cultura organizacional, creatividad y gestión considerados componentes de esencial importancia para la generación de los emprendimientos y que facilitan mejorar los resultados económicos para los pequeños, medianos y grandes emprendedores. La cultura es un componente fundamental del proceso de socialización y del conocimiento que se requiere para alcanzar ventajas que le permitan competir y tiene como objetivo describir acercamientos acerca de la cultura, creatividad y gestión para la generación de emprendimientos, para ello se aplicó metodología descriptiva, la que permitió realizar indagaciones mediante una ficha de observación que se realizó a los resultados presentados en la matriz de la feria de emprendimiento, llegando a las conclusiones de que la capacitación y acompañamiento a los emprendedores, genera confianza para empezar con los negocios aportando de esta forma a la economía familiar y a la universidad en sus procesos académicos.

PALABRAS CLAVES: Cultura, Creatividad, Emprendimiento, Gestión, Motivación

\begin{abstract}
This work focuses on the review of the scientific basis of the organizational culture, creativity and management considered components of essential importance for the generation of enterprises and that facilitate the improvement of economic results for small, medium and large entrepreneurs. Culture is a fundamental component of the process of socialization and knowledge and is required to achieve advantages that allow it to compete. Its objective is to describe some approaches about culture, creativity and management for the generation of entrepreneurship, for this a descriptive methodology was applied, which allowed ... to make observations through an observation sheet in which the results presented in the matrix were made of the entrepreneurship fair, reaching the conclusions that training and support for entrepreneurs, generates confidence to start with family businesses, thus contributing to the family economy and the university in their academic processes.
\end{abstract}

KEYWORDS: Culture, Creativity, Learning, Management, Motivation

\footnotetext{
${ }^{1}$ Ing comercial - Máster en Administración de Empresas, Universidad Técnica de Babahoyo, Ecuador. Correo electrónico: mhuilcapi@utb.edu.ec

${ }^{2}$ Ing comercial - Máster en Administración de Empresas, Universidad Técnica de Babahoyo, Ecuador. Correo electrónico: mmazacon@utb.edu.ec

${ }^{3}$ Ing comercial - Máster en Administración de Empresas, Universidad Técnica de Babahoyo, Ecuador. Correo electrónico: lcaicedo@utb.edu.ec
} 


\section{INTRODUCCIÓN}

La motivación según (Huilcapi Masacón, Castro López, \& Jácome Lara, 2017) señala que el ser humano tiene dos tipos de necesidades, una de ellas le permite evadir o defenderse, la otra le permite satisfacer sus carencias, cuando lo ha logrado se siente motivado para hacer sus actividades. De igual manera Munch (2010), define así a la motivación: "Es una de las labores más importantes de la dirección, a la vez que es la más compleja, pues, por medio de ésta se logra que los empleados ejecuten el trabajo con responsabilidad y agrado, de acuerdo con los estándares establecidos, además de que es posible obtener el compromiso y la lealtad del factor humano". Según Abraham Maslow la motivación tiene jerarquías, Ver fig. 1

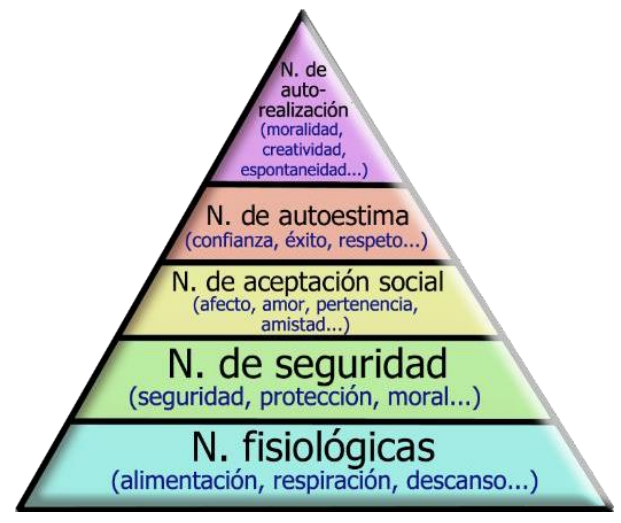

Figura 1. Pirámide de necesidades, según (A. Maslow).

Estas mismas necesidades pueden generar en el ser humano motivación para emprender. El emprendimiento se lo define como una solución a los problemas sociales, y económicos de los pueblos, la grave crisis económica que la población atraviesa obliga a buscar alternativas para superar la falta de recursos económicos en el hogar, según (Martínez, 2018), citado por (Huilcapi Masacon, Mora Aristega, \& Castro López, 2018), hablar del emprendimiento como opción de vida para la generación de ingresos, es encontrar dos tipologías: los emprendimientos por oportunidad y los que se generan por necesidad. Los visionarios identifican las oportunidades y las aprovechan, los que emprenden por necesidad se enfrentan al mercado sin análisis ni estudios previos y en ocasiones tienen éxitos.

La cultura comprende el patrón general de conductas, creencias y valores compartidos por los miembros de una organización (Salazar Estrada, Guerrero Pupo, Machado Rodríguez, \& Cañedo Andalia, 2009). El mundo globalizado de hoy frente a los procesos económicos acelerados requiere dinamismo en los procesos, de una comunicación que fluya en doble vía, hacer uso de los sistemas de la información y comunicación, según (Parra, 2007), además requiere que los emprendedores posean ciertas características, que según (Suleiman) son necesarias para asumir con capacidad de gestión los emprendimientos, estas son:

- Disposición para asumir riesgos

- Imaginación

- Seguridad de sí mismo

- Persistencia

- Capacidad para adaptarse 
Es importante determinar la relación entre la cultura, creatividad y gestión para generar emprendimientos viables que permitan la creación de microempresas para el desarrollo de los países. Desde el momento que las Instituciones de Educación Superior realicen actividades que incentiven la creatividad y se formen hábitos para generar emprendimientos tanto en estudiantes como en los docentes y empleados se van a generar microempresas en el país, las mismas que van a contribuir al desarrollo económico de la población.

La misión de las Instituciones de Educación Superior es formar profesionales en las diferentes áreas que ofertan las IES, esa formación debe incluir la generación de emprendimientos mediante programas, charlas, capacitaciones, ferias, concursos de emprendimiento que moti|ven y fomenten la creatividad y hábitos indispensables para la generación de emprendimientos.

Es muy importante la articulación de los diferentes sectores públicos y privados para la inversión y difusión de los proyectos de emprendimientos, que generan los estudiantes mediante ferias, casa abierta, promociones de productos en cada una de las IES, con la finalidad que se logre el interés por parte de los estudiantes en la participación y desarrollo de ideas innovadoras para el beneficio de la sociedad en general.

\section{Cultura del emprendimiento.}

La cultura del emprendimiento es el entorno que favorece el desarrollo de proyectos emprendedores, entendidos como procesos de fundación de actividades, proyectos, empresas lucrativas o no, de índole económica, social, política, o también se refiere a renovación o reingenierías de procesos existentes (Proaño, 2014). Destacando que en el entorno educativo en el cual se desempeñan los estudiantes, se establezcan mecanismos para favorecer el emprendimiento desde un ámbito cognitivo y metacognitivo, a fin de que los estudiantes tengan facilidad en el desarrollo de los proyectos.

Aunque en los actuales momentos el mundo enfrenta una pandemia generada por el COVID-19, la cual ha causado una crisis económica a nivel mundial, por lo que es necesario implementar una cultura del emprendimiento en la IES, con la finalidad de que los estudiantes, rompan los paradigmas que se han implementado desde la familia, que establece la búsqueda de empleo y no la generación del mismo, al romper los paradigmas se acortan las brechas del desempleo, de la desigualdad, fortaleciendo el núcleo familiar, la economía del hogar, de la comunidad y su entorno.

Según, (Drucker,1985), "El emprendimiento es maximizar las oportunidades, es decir, la efectividad y no la eficiencia; es la esencia del trabajo" La Cultura del emprendimiento y la formación del Emprendedor, es un rasgo de comportamiento que puede o no verificarse en ciertos tipos de individuos y organizaciones. Obviamente, este perfil es más frecuente observarlo en las empresas más pequeñas y nuevas y en las personas de edad mediana que en las personas mayores; quizás porque la típica actitud "juvenil" es ser muy sensible a las condiciones que pueden favorecer su desarrollo. Lo que no excluye que personas mayores y organizaciones "adultas" puedan convertirse en emprendedoras, si algo les provoca hacer y su capacidad les permita reaccionar, diferente a la inercia de su historia. (Proaño, 2014). Para el desarrollo de la cultura del emprendimiento en las IES, es necesario definir, estrategias administrativas, estrategias didácticas, seminarios de capacitación, desarrollo de eventos científicos (casa abierta, ferias de emprendimientos), que incentiven la participación de los estudiantes.

\section{CREATIVIDAD}

Vygotsky (1981) consideraba que la creatividad existe potencialmente en todos los seres humanos, y es susceptible de desarrollar; o sea, que no es privativa de los genios, sino que está presente en cualquier ser humano que imagine, transforme o cree algo por insignificante que sea, en comparación con las grandes personalidades creativas de la historia. Graham Wallas (1926), en su obra "El arte 
del pensamiento", fue pionero en la presentación de un modelo definiendo el proceso creativo. En el modelo de Wallas, se agrupaba los enfoques creativos para explicar este proceso en cinco etapas:

Preparación: Se identifica como el primer estadio del proceso creativo, etapa que identifica el problema de interés, se enfoca en la mente y explora las dimensiones de dicho problema.

Incubación: Etapa de tensión emocional, se interioriza mentalmente el problema en el hemisferio derecho y parece que nada pasa externamente.

Intimación: La persona creativa, activa esta característica intuye que existe una solución al problema y busca aproximarse a ella en su mente.

Iluminación o insight: Se da cuando aparece en la mente de cada persona la solución y surge la creatividad en forma de idea, es decir brota del procesamiento mental interior una solución consciente y real.

Verificación: cuando la idea es conscientemente, verificada, validada, elaborada y luego aplicada para solucionar el problema.

Para Arnold, (1964), todo proceso creativo es análogo al proceso de solución de un problema; se trabaja con la información que se tiene a mano, se ponen en juego las experiencias anteriores, se las combina y traslada a las nuevas estructuras, que en su nueva configuración resuelven un problema, el cual satisface alguna necesidad del individuo (Landau, 1987). La creatividad es necesaria para una infinidad de aspectos de la vida diaria, el desarrollo de la creatividad a través de la educación es necesaria por su importancia transversal en la interacción con el entorno económico, social, humano, laboral.

El término anglosajón entrepreneurship está estrechamente relacionado con el vocablo francés entrepreneur, que aparece a principios del siglo XVI. En economía, negocios, finanzas, etc., tiene el sentido más específico de ser aquel individuo que está dispuesto a asumir un riesgo económico. Desde este punto de vista el término se refiere a quien identifica una oportunidad y organiza los recursos necesarios para ponerla en marcha (Liñan, 2004), y satisfacer esa necesidad identificada.

Las IES, deberán ajustar sus modelos educativos a formar profesionales capaces de generar emprendimiento, para luego generar trabajo a otras personas, es un reto que se deberá proponer tanto para los estudiantes como para los docentes, dado que dicha cultura de emprendimiento demanda más esfuerzo y preparación de los involucrados. De ahí la importancia de formar emprendedores que se encuentren en la capacidad de identificar ideas de negocios, evaluarlas e implementarlas, con una base técnica-científica, lo cual les permitirá tener una mayor probabilidad de éxito.

Innovación sería la utilización de una nueva tecnología, hasta entonces desconocida; pero también la aplicación de una tecnología ya conocida que hasta la fecha no se venía usando en esa actividad. Del mismo modo, la decisión de exportar también constituiría una innovación. En todo caso, el rasgo común de todas estas actuaciones es su carácter no rutinario, de forma que podrían corresponderse con lo que el profesor Santos (2001), denomina comportamientos dinamizadores.

\section{MATERIALES Y MÉTODOS}

Los programas de emprendimiento se han constituido en una herramienta de los gobernantes para aumentar la generación de ingresos y de empleo productivo, de allí que, a nivel de entidades gubernamentales, corresponde a ellas diseñar políticas locales asociadas al fortalecimiento de las PYMES, las mismas que desempeñan un papel creciente de la economía y contribuyen a la generación de empleo, al desarrollo de la economía y la sociedad en general, dinamizando así las cadenas productivas (Marleny Cardona A. Luz Dinora Vera, 2016). 
Con respecto a la gestión, vale destacar que la responsabilidad social corporativa ayuda a las organizaciones a ser socialmente responsables ante las sociedades y las partes interesadas con respecto a sus diferentes impactos en comunidades como la económica, social y ambiental (Qureshi., 2019).

\section{METODOLOGÍA}

La metodología utilizada fue la investigación descriptiva, la que permitió realizar observaciones utilizando como instrumento una guía de observación a los informes presentados por la comisión de feria de emprendimientos la misma que permitió realizar puntualizaciones de rasgos referentes al caso en estudio.

\section{RESULTADOS Y DISCUSIÓN}

El presente trabajo se lo realizó en base a los proyectos de emprendimiento presentados en las ferias realizadas en la Facultad de Ciencias de la Salud de la Universidad Técnica de Babahoyo, donde se determinó que desde el año 2017 hasta el año 2019 el número de proyectos presentados como el número de estudiantes ha ido en aumento, lo que conlleva a ir fomentando la cultura, incentivando la creatividad de emprendimiento en estudiantes, de igual forma generar una gestión factible para la presentación, así como para su ejecución de los emprendimientos. El número de asistentes que acudieron a las ferias fue en un alto porcentaje de diversas instituciones educativas de nivel medio de la ciudad de Babahoyo, donde observaron, se interesaron y les llamó la atención la creatividad de los estudiantes universitarios a través de sus proyectos de emprendimiento en la facultad objeto de estudio.

Tabla 1

Estadísticas de la Feria de Emprendimientos FCS. UTB.

\begin{tabular}{|c|c|c|c|c|c|}
\hline Años & Feria & Carreras & $\begin{array}{l}\text { No } \\
\text { Estudiantes } \\
\text { Practicantes }\end{array}$ & Asistentes & $\begin{array}{l}\text { No Pryectos } \\
\text { presentados }\end{array}$ \\
\hline 2017 & I Feria Emprendimiento & $\begin{array}{l}\text { Optometría } \\
\text { Enfermería } \\
\text { Obstetricia } \\
\text { Terapia Respiratoria } \\
\text { Nutrición }\end{array}$ & 50 & 740 & 9 \\
\hline 2018 & II Feria Emprendimiento & $\begin{array}{l}\text { Optometría } \\
\text { Enfermería } \\
\text { Obstetricia } \\
\text { Terapia Respiratoria } \\
\text { Nutrición }\end{array}$ & 45 & 740 & 12 \\
\hline 2019 & $\begin{array}{l}\text { III Feria } \\
\text { Emprendimiento }\end{array}$ & $\begin{array}{l}\text { Optometría } \\
\text { Enfermería } \\
\text { Obstetricia } \\
\text { Terapia Respiratoria } \\
\text { Fisioterapia } \\
\text { Nutrición }\end{array}$ & 72 & 520 & 18 \\
\hline
\end{tabular}

Del análisis se puede determinar que del primer año de Feria 2017, al segundo año hubo un crecimiento del 133\%; en el 2019 en cambio el crecimiento comparado con el primer año (2017) fue 
del 200\%, y con el segundo año 2018, hubo un crecimiento del 150\%. Evidenciando que los estudiantes si se han interesado por la generación de emprendimientos.

\section{Discusión.}

La cultura organizacional, creatividad y gestión considerados componentes de esencial importancia para la generación de los emprendimientos y que facilitan mejorar los resultados económicos para los pequeños, medianos y grandes emprendedores y como misión fundamental de las universidades formar profesionales emprendedores.

\section{CONCLUSIÓN}

Corresponde a la Universidad realizar capacitación y acompañamiento a los emprendedores, pues esto genera confianza para empezar con los negocios aportando de esta forma a la economía familiar y a la universidad en sus procesos académicos hasta llegar a patentar los emprendimientos.

Generación de ferias de emprendimiento, que promueven de forma eficaz la cultura en los estudiantes para el aprendizaje en el desarrollo de proyectos de emprendimiento.

Innovación en proyectos de emprendimientos que permitan generar productos y/o servicios creativos para solución de problemas económicos de las comunidades.

\section{REFERENCIAS}

Huilcapi Masacon, M. R., Mora Aristega, J. E., \& Castro López, G. (2018). Masacon, M. R. H., Aristega, J. E. Emprendimiento opción para reactivar la economía y el desarrollo sostenible en comuna Santa Elena. Masacon, M. R. H., Aristega, J. E. M., \& López, G. A. C. (2018). Emprendimiento opción para reactivar la economía y el desarrollo Killkana sociales: Revista de Investigación Científica., 59-64.

Marleny Cardona A. Luz Dinora Vera, J. T. (2016). LAS Dimensiones del emprendimiento empresarial: La experiencia. Documentos de trabajo de Economía y Finanzas.

Parra, D. Q. (2007). Comunicación, clima y cultura organizacional para la gestión del conocimiento. Pymes metalmecánicas de Cali. Universidad. Empresa, Bogotá (Colombia), 9-36.

Proaño, L. F. H. (2014). La cultura del emprendimiento y su formación. Alternativas, 15(1), 46-50.

Qureshi., M. H. (2019). Impacto de la responsabilidad social corporativa en la lealtad de los clientes: el papel mediador de la confianza de los clientes. Dilemas Contemporáneos: Educación, Política y Valores.

Salazar Estrada, J., Guerrero Pupo, J., Machado Rodríguez, Y., \& Cañedo Andalia, R. (2009). Clima y cultura organizacional: dos componentes esenciales en la productividad laboral. ACIMED., 67-75.

Proaño, L. F. H. (2014). La cultura del emprendimiento y su formación. Alternativas, 15(1), 46-50.

Druker, Peter (1998), Su visión sobre La Administración, la Organización Basada en la Información, La Economía y La Sociedad, Bogotá, Editora Norma

Bhidé, Armar V. (1999): “Cómo Elaboran los emprendedores estrategias que funcionan”; Harvard.

CONEA (2003), La Calidad de la Universidad Ecuatoriana: Principios, Características y Estándares de Calidad, Asistencia Técnica UNESCO/IESALC, Quito.

Varela Rodrigo (2004), La Educación, la Universidad y la Cultura Empresarial en América Latina: La Experiencia de la Universidad ICESI, http://www. iadb.org/sds/doc/conf.6.24.04.varela.pdf

Rojas Caicedo, G., Quintero, L., Pertuz Peralta, V., \& Navarro Rodríguez, A. (2015). Estrategias para el fomento de la cultura de emprendimiento universidades de Valledupar, Colombia. Revista Educación Y Desarrollo Social, 10(1), 38-57. https://doi.org/10.18359/reds.1448. 
Vigotsky, L. (1981). The genesis of higher mental funtions. En Wertsch, J. V. (1981). The concept of activity in Soviet Psychology. New York: Sharpe.

Wallas, G. (1926). The Art of Thought. New York: Harcourt Brace

Landau, E. (1987). El vivir creativo: teoría y práctica de la creatividad. Barcelona: Editorial Herder.

Liñán, F. (2004). Educación empresarial y modelo de intenciones. Formación para un empresariado de calidad. Sevilla: Universidad de Sevilla.

Santos, F.J. (2001). La calidad del empresario sevillano. Sevilla: Siglo XXI

\section{CONFLICTOS DE INTERESES}

Los autores no refieren conflictos de intereses 